\title{
Prevalencia de rinitis alérgica en pacientes pediátricos que acuden al servicio de emergencia del Hospital Nacional Cayetano Heredia por crisis de asma.
}

PEREZ LU Julio Enrique , CENTENO HUAMAN Jorge* , CHIARELLA ORTIGOZA Pascual** , PEREZ LU Luis Enrique, SIALER CHAVEZ Salvador Teodoro.

\begin{abstract}
SUMMARY
Objective: To know prevalence of allergic rhinitis (AR) in children with acute event of asthma who go to emergency room of the Hospital Nacional Cayetano Heredia. To determine prevalence of acute rhinitis episode, and prevalence of diagnosis of $\mathrm{AR}$ before patients come to emergency. Material and Methods: It is a descriptive traverse study. The size sample was 164 children $(\alpha=95 \% ; \beta=5 \%)$. We interviewed parents with a questionnaire to find symptoms of $A R$ and acute nasal symptom. This questionnaire was used in ISAAC study and a prevalence work about AR made in our country. Results: 169 children from 2 to 14 years went for acute event of asthma to HNCH's emergency, between March and June of 2002. The average age was 7. $59 \%$ were moderate asthmatics, with an average clinic score of acute episode of asthma in $5.2 .82 .8 \%$ of the patients had nasal symptoms in the moment they went to the hospital. $83.4 \%$ had nasal symptoms in the last 12 months, not associated to cold or flu. We found a prevalence of AR of 54.4\% $10.7 \%$ of patients had previous diagnosis of AR, before going to emergency. Conclusion: Coexistence of $\mathrm{AR}$ and asthma is frequent in our means. It is necessary to recognize it for a better handling. There is poor information in parents about this allergy and its relationships to asthma. ( Rev Med Hered 2003; 14: 111-116 ).
\end{abstract}

KEY WORDS: Allergic rhinitis, acute event of asthma.

\section{RESUMEN}

Objetivo: Conocer la prevalencia de rinitis alérgica (RA) en los niños que acuden por crisis de asma a la de Emergencia del Hospital Nacional Cayetano Heredia. Determinar la prevalencia de cuadro activo de rinitis y de pacientes con diagnóstico previo de RA en el grupo de estudio. Material y Método: Es un estudio transversal, descriptivo. El tamaño muestral fue de 164 niños $(\alpha=95 \% ; \beta=5 \%)$. Se realizó un cuestionario mediante una entrevista directa a los padres para encontrar síntomas de RA y síntomas nasales agudos. Se empleó un cuestionario estandarizado internacionalmente y utilizado en un estudio epidemiológico en nuestro medio. Resultados: Ingresaron 169 niños de 2 a 14 años, que acudieron por crisis de asma a la sala de Emergencia del HNCH, entre marzo y junio de 2002. La edad promedio fue de 7 años. El 59\% fueron asmáticos moderados, con un puntaje clínico promedio de crisis de ASMA de 5.2. El 82.8\% de los pacientes tuvieron síntomas nasales en el momento que acudieron al hospital. El 83.4\% presentaron síntomas nasales en los últimos 12 meses, no asociados a resfrío o gripe. Se encontró una prevalencia de RA de 54.4\%. El 10.7\% tuvieron diagnóstico previo de RA, siendo un valor menor al esperado. Conclusión: La coexistencia de RA y asma es frecuente en nuestro medio. Es necesario reconocerla para un mejor manejo. Hay una falta de conocimiento e información de los padres sobre esta alergia y su relación con el asma.( Rev Med Hered 2003; 14:111-116).

PALABRAS CLAVES : Rinitis alérgica, crisis asma.

\footnotetext{
* $\quad$ Médico Cirujano, especialidad en otorrinolaringología.Universidad Peruana Cayetano Heredia. Departamento de Cirugia, Hospital Nacional Cayetano Heredia.

** Médico Cirujano, especialidad en neumología pediátrica. Universidad Peruana Cayetano Heredia.Departamento de Pediatria, Hospital Nacional Cayetano Heredia.
} 


\section{INTRODUCCION}

La disfunción de la vía aérea alta y baja coexiste con frecuencia. Además, dentro de su fisiopatología tienen algunos elementos en común. Se ha observado que los pacientes asmáticos con rinitis alérgica (RA), que llevan tratamiento para rinitis tienen menor riesgo de visitas al servicio de emergencia y hospitalizaciones por crisis de asma, que aquellos que no lo reciben (1).

Muchos estudios epidemiológicos han descrito que la coexistencia de RA y asma es muy frecuente. Sin embargo, estos datos son muy variables a nivel mundial, dependiendo del lugar y cómo se haya realizado la investigación. En este sentido, la literatura señala valores de prevalencia de RA en pacientes con asma que van del $78 \%$ hasta el $95 \%$. Los estudios que señalan esto han sido realizados en niños, adolescentes y la población general, principalmente en países occidentales (1-5).

Ambas entidades ocasionan grandes gastos en la asistencia sanitaria, así como la pérdida de millones de días laborales y escolares. De todos los grupos etarios, los niños y adultos jóvenes son los más afectados (3).

En diciembre de 1995 se reunió un grupo de expertos en la Organización Mundial de la Salud (OMS) en Ginebra para elaborar una guía de recomendaciones para el diagnóstico de la rinitis y otros procesos inflamatorios interrelacionados. Así surgió ARIA (Allergic Rhinitis and its Impact on Asthma). La iniciativa de este grupo ha sido lanzada en febrero del 2001, en Tokio. Dentro de sus recomendaciones propone que se considere a la RA como factor de riesgo para asma (6).

Actualmente no hay consenso en cuanto al tipo de relación que existe en la coexistencia de asma y RA, solamente se han establecido algunas hipótesis acerca de los mecanismos fisiopatológicos (5).

Realizar un trabajo en pacientes pediátricos, nos va a permitir investigar sobre uno de los grupos etarios más afectado. Las conclusiones obtenidas podrán ser aplicadas para la población de pacientes que acuden al servicio de emergencia de pediatría del Hospital Nacional Cayetano Heredia (HNCH). Con estos datos podemos darnos una idea de que es lo que podría estar sucediendo en otros sectores de Lima. Este es un paso inicial en nuestro medio, en el estudio epidemiológico de la asociación de estas dos patologías.

Nuestro objetivo principal fue conocer la prevalencia de RA en los niños con crisis de asma que acuden al servicio de emergencia del $\mathrm{HNCH}$, en 2002. Por otro lado, quisimos determinar la prevalencia de pacientes con cuadro activo de rinitis y de pacientes con diagnóstico previo de RA en el grupo de estudio.

\section{MATERIAL Y METODOS}

Este es un estudio transversal de carácter descriptivo. Según los reportes estadísticos del servicio de emergencia pediátrica del $\mathrm{HNCH}$ se atienden aproximadamente 1000 niños por crisis de asma trimestralmente. Según la literatura extranjera, la prevalencia de RA en pacientes asmáticos va del 78 al 95\% (1-5). Para nuestros cálculos tomamos $85 \%$ como valor de referencia. Se calculó 164 niños como tamaño de muestra, con un $95 \%$ de confianza y $5 \%$ de precisión.

Se evaluaron los niños con crisis de asma que acudieron a la emergencia pediátrica del $\mathrm{HNCH}$, entre mediados de marzo y junio de 2002. El conteo y tipo de muestreo fue por conveniencia.

Se empleó el puntaje clínico de Bierman y Pierson corregido por Tal para establecer la severidad de la crisis de los pacientes en el momento que llegan a emergencia (7).

Se aplicó un cuestionario (ver Tabla $\mathrm{N}^{\circ} 1$ ) mediante una entrevista directa a los padres, para conocer la presencia de síntomas nasales y de RA en el momento en que el paciente acude al hospital. Para establecer el diagnóstico de RA se utilizó un cuestionario estandarizado internacionalmente que fue empleado en un estudio de prevalencia de RA, realizado en nuestro medio $(8,9)$. Para evaluar la severidad de los síntomas nasales se utilizó un puntaje clínico utilizado en un ensayo clínico sobre pacientes con RA (10).

Tabla $N^{\circ} 1$ Puntaje clínico de los síntomas nasales

\begin{tabular}{|c|c|c|c|c|c|}
\hline \multicolumn{6}{|c|}{ PUNTAJE DE LOS SÍNTOMAS NASALES: (10) } \\
\hline & 0 & 1 & 2 & 3 & \multirow{6}{*}{$\begin{array}{l}0=\text { No hay síntoma } \\
1=\text { El síntoma no es un } \\
\text { problema real para el } \\
\text { paciente (LEVE) } \\
2=\mathrm{El} \text { síntoma es } \\
\text { considerado un } \\
\text { problema para el } \\
\text { paciente pero no } \\
\text { interfiere con sus } \\
\text { actividades diarias } \\
\text { (MODERADO) } \\
3=\text { El síntoma produce } \\
\text { gran interferencia con } \\
\text { la actividad diaria del } \\
\text { paciente (SEVERO) }\end{array}$} \\
\hline Rinorrea & & & & & \\
\hline $\begin{array}{l}\text { Congestión } \\
\text { Nasal }\end{array}$ & & & & & \\
\hline Estornudos & & & & & \\
\hline Prurito & & & & & \\
\hline \multicolumn{5}{|c|}{$\begin{array}{l}\text { TOTAL: } \\
\text { POR LO MENOS RINORREA y/o } \\
\text { CONG. NASAL con puntaje } \geq 2 \text { y } \\
\text { PUNTAJE TOTAL } \geq 5 \text { PARA SER } \\
\text { CONSIDERADO COMO } \\
\text { SINTOMATOLOGÍA } \\
\text { MODERADA A SEVERA }\end{array}$} & \\
\hline
\end{tabular}


Cuestionario estandarizado utilizado en el estudio para evaluar la presencia de rinitis alergica $(8,9)$

¿Su niño ha tenido estornudos, secreción nasal y se le ha tupido la nariz alguna vez en su vida, no asociados a resfrío o gripe?

¿Su niño ha tenido estornudos, secreción nasal y se le ha tupido la nariz en los últimos 12 meses, no asociados a resfrío o gripe?

¿Aparte de los síntomas descritos, a su niño además le picaban y lloraban los ojos en los últimos 12 meses?

¿En qué meses del año se han presentado los síntomas antes descritos con más frecuencia; no asociados a resfrío o gripe?

¿Cómo influyeron los síntomas antes descritos en la vida diaria de su niño?

¿Su niño ha tenido el diagnóstico de rinitis alérgica?

Si los padres respondían afirmativamente la pregunta 1 , continuaban con el cuestionario. Si la respuesta era negativa se consideraba que el paciente no tenía rinitis y se finalizaba la entrevista. Si la segunda pregunta era afirmativa, se calificaba al paciente como portador de una historia compatible con rinitis no infecciosa y se pasaba a la siguiente pregunta. Si era negativa se descartó la posibilidad de la presencia de rinitis en el paciente. Si los padres respondían a las tres primeras preguntas de forma afirmativa se estableció el diagnóstico de RA. En caso de que solamente la pregunta 1 y 2 fueran afirmativas, se consideró al paciente como portador de una historia sugestiva de rinitis no alérgica, no infecciosa. Luego de haber respondido a las primeras 3 preguntas se continuó resolviendo el cuestionario hasta el final.

\section{Criterios de inclusión}

a) Pacientes $>$ de 2 y $<14$ años (sólo los menores de 14 años son atendidos en el servicio de emergencia pediátrica del $\mathrm{HNCH}$ ), de ambos sexos.

b)Pacientes con diagnóstico previo de asma, que acudan al servicio de emergencia pediátrica del $\mathrm{HNCH}$ por tos persistente, sibilancias y dificultad respiratoria.

c) Pacientes sin diagnóstico previo de asma, que en alguna oportunidad hayan requerido usar bronco dilatadores que acudan al servicio de emergencia de pediatría del $\mathrm{HNCH}$ por tos persistente, sibilancias y dificultad respiratoria.

\section{Criterios de exclusión}

Paciente que acuda por primer episodio de broncoespasmo.

\section{Análisis Estadístico}

Se utilizó el programa Epiinfo 6.0 para determinar las medidas de frecuencias.

\section{RESULTADOS}

Ingresaron 169 niños, que acudieron por crisis de asma al Servicio de Emergencia del $\mathrm{HNCH}$, desde mediados de marzo hasta junio de 2002. La edad promedio fue de 7.4 años, y la mediana de 7 años (DS: 3.05). La relación niño/niña fue de 7/6. El 84.6\% de los pacientes tuvo diagnóstico previo de ASMA. El $59.2 \%$ fueron pacientes asmáticos moderados. El puntaje clínico promedio de crisis de ASMA de Bierman y Pierson fue de 5.21 y la mediana de 5 (DS: 1.47).

El 82.8\% de los pacientes tuvieron síntomas nasales en el momento que acudieron al hospital. Este vendría a ser el grupo de pacientes con un cuadro activo de rinitis. El 56.8\% de ellos tuvo un puntaje moderado/ severo $(\mathrm{M} / \mathrm{S})$, según la escala utilizada. El valor promedio de éste fue de 5.77 y la mediana de 6 (DS: 2.25). El grupo (M/S) constituye el $68 \%$ del grupo que presentaron síntomas nasales agudos (ver Tabla $\mathrm{N}^{\circ}$ 2).

El 83.4\% señaló haber presentado síntomas nasales, no asociados a resfrío o gripe, en algún momento de su vida. El mismo porcentaje se encontró que presentaron síntomas nasales, no asociados a resfrío o gripe, en los últimos 12 meses (ver Tabla $\mathrm{N}^{\circ} 2$, gráfico $\mathrm{N}^{\circ} 1$ ). Además, se encontró que la prevalencia de RA en el grupo de estudio fue de $54.4 \%$ y de rinitis NO alérgica (RNA) del 29\%. Dentro del grupo de pacientes con rinitis, los que tienen RA representan el $65.2 \%$ y el de RNA $34.8 \%$.

\section{Tabla N $\mathbf{N}^{\mathbf{2}}$. Resultados del cuestionario de} evaluación.

\begin{tabular}{|lcc|}
\hline & $\mathrm{n}$ & Porcentaje \\
\hline $\begin{array}{l}\text { Síntomas nasales al llegar a la } \\
\text { emergencia }\end{array}$ & 140 & $82.8 \%$ \\
$\begin{array}{l}\text { Severidad de síntomas nasales } \\
\text { Leve } \\
\text { Moderado/Severo }\end{array}$ & 45 & $26.6 \%$ \\
$\begin{array}{l}\text { Presencia de síntomas nasales } \\
\text { alguna vez en la vida, no asociado } \\
\text { a resfrío o gripe }\end{array}$ & 96 & $56.8 \%$ \\
$\begin{array}{l}\text { Presencia de síntomas nasales, no } \\
\text { asociado a resfrío o gripe, en el } \\
\text { último año }\end{array}$ & 141 & $83.4 \%$ \\
$\begin{array}{l}\text { Pacientes que acudieron con } \\
\text { diagnóstico previo de RA }\end{array}$ & 141 & $83.4 \%$ \\
\hline TOTAL (N) & 18 & $10.7 \%$ \\
\hline
\end{tabular}




\section{Gráfico $\mathbf{N}^{0} 1$. Distribución de los tipos de rinitis en} el total del grupo de estudio.

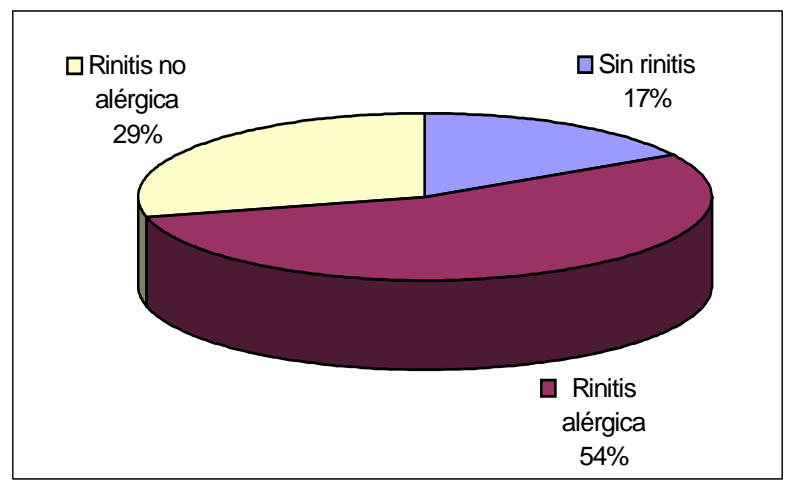

Los niños que acudieron a la emergencia con diagnóstico previo de RA constituyeron el 10.7\% del grupo total de pacientes. Si lo comparamos con el grupo de pacientes con rinitis (no infecciosa) sola, el valor sería de $12.8 \%$. Dentro del grupo de niños con RA vendría a ser el $19.6 \%$.

A la pregunta de "¿En qué meses del año se le han presentado los síntomas antes descritos con más frecuencia; no asociados a resfríos o gripe?", la mayoría respondió que en invierno y de esta estación el mes de julio como mes de mayor frecuencia de síntomas nasales (45\%, de todos los pacientes). Sólo 32 pacientes señalaron que la ocurrencia de estas molestias era por igual a lo largo de todo el año, y esto corresponde al $22 \%$ de los pacientes con rinitis (Rinitis alérgica persistente) (6).

\section{DISCUSIÓN}

Este estudio trata de establecer un estimado epidemiológico de la asociación entre RA y asma, en población infantil. En este caso, se eligió estudiar al grupo de niños que acuden por crisis de asma a la emergencia de $\mathrm{HNCH}$. Realizar esta investigación no es lo mismo que hacerlo en el grupo total de asmáticos, porque generalmente quienes llegan a emergencia por crisis de asma son pacientes con una historia de asma moderada a severa. Muy pocos son los pacientes con asma leve que desarrollan una crisis tan severa que requiere un manejo en el hospital.

Los pacientes que acuden a este centro no son de todos los distritos de Lima. La mayoría pertenece al cono norte de la ciudad, la cual es una zona de tipo urbano y urbano-marginal, quienes en su mayoría son de un nivel socioeconómico bajo.Tener en cuenta el lugar de procedencia es importante para la interpretación de los resultados, ya que hay factores ambientales y sociales que promueven el desarrollo y mantenimiento del cuadro inflamatorio correspondiente a la rinitis y el asma.

En este estudio se han empleado 2 instrumentos de evaluación epidemiológica. Uno de ellos asignó un puntaje clínico según la presencia de síntomas nasales en el momento que los pacientes acuden a la emergencia. Este puntaje ha sido utilizado en un ensayo clínico realizado en adultos con RA. De esta manera pudimos realizar una evaluación objetiva y cuantitativa (8).

Para determinar si el paciente era portador de una historia sugerente de RA se usó un cuestionario estandarizado internacionalmente. Este fue diseñado para el estudio ISAAC (Internacional Study of Asthma and Allergies in Childhood) y se ha utilizado en nuestro medio, en un estudio epidemiológico realizado en niños con RA publicado en 1997. En este estudio Ruiz y col. valoraron la prevalencia de RA en la población escolar del Distrito de Santiago de Surco-Lima. Ellos encontraron una prevalencia del $11.1 \%$ de RA en un total de 3158 alumnos entrevistados directamente, evaluando niños de 13 a 14 años aplicando directamente la encuesta $(8,9)$.

Estos estudios mencionados enfrentaron directamente el cuestionario a los niños. El grupo etario evaluado fueron niños de 6 a 7 años y niños de 13 a 14 años. En este trabajo el cuestionario fue aplicado mediante una entrevista con a los padres de estos niños cuyas edades estaban comprendidas entre los 2 y 14 años. Se decidió hacer éste cambio en la aplicación del cuestionario por considerarse que era más factible esta forma de evaluación, dadas las dificultades que implicaban el desarrollo del mismo directamente aplicado a los pacientes en un servicio de emergencia $(6,8,9)$.

Con el puntaje de síntomas nasales determinamos la presencia de un cuadro agudo de rinitis, más no de la naturaleza de ésta. El $82.8 \%$ de los pacientes presentaron estos síntomas, siendo la mayoría de estos de un grado moderado/severo. Por ser un estudio descriptivo, no podemos esclarecer con detalle la importancia de este hallazgo. Cabría realizar estudios más profundos para ver si existe alguna relación y de que tipo, entre estos datos y las crisis o el tipo de asma.

El cuestionario utilizado para calificar a los pacientes como portadores o no de RA nos permite identificar con seguridad a niños con RA. Algunos consideran que es un buen instrumento para el estudio de datos epidemiológicos $(10,15)$. A pesar de ello puede ser cuestionado, ya que no se hace uso de exámenes auxiliares para establecer un diagnóstico. El 
diagnóstico diferencial de los tipos de rinitis es muy amplio. Sin embargo, dichas pruebas de laboratorio son de muy baja sensibilidad, con una confiabilidad muy variable y alto costo económico. Estas mismas no son de uso rutinario en la consulta diaria para el diagnóstico de $\operatorname{RA}(10,15,16)$.

El estudio SCARPOL, validó el cuestionario ISAAC en una población de escolares con rinitis en Suecia (n =2954) comparado con la prueba cutánea de reacción. En esta evaluación se determinó una especificidad del cuestionario del 77.5 al $97.6 \%$, pero una sensibilidad baja (2.6 al 42.7\%) (6). Por la alta especificidad de este cuestionario hay bastante seguridad en la certeza en el diagnóstico. Sin embargo, como ya se dijo, en este estudio no se ha aplicado estrictamente como se diseñó, en relación a la edad de los pacientes en quienes se aplicó la encuesta y la forma de aplicación. Por lo tanto, no sabemos si algunos casos que hemos registrado como casos de rinitis se han confundido con infecciones virales por ejemplo, principalmente en los niños más pequeños.

Hemos hallado que la prevalencia de rinitis fue de $83.4 \%$. A pesar de ello, coincide con las cifras de otros estudios de prevalencia de RA en pacientes asmáticos (1-5).

También hemos estimado un valor de prevalencia de RA en este grupo de 54.4\%. Esta cifra es menor a la encontrada en la literatura. Quizás se deba a que la prevalencia que hemos encontrado es la mínima confiable, porque al ir uniendo los síntomas con el diagnóstico se subestima la cifra real, pero a la vez hace que este dato sea más específico. Probablemente el verdadero porcentaje de RA en estos pacientes sea mayor, $\mathrm{y}$ tal vez se acerque al de rinitis que hemos calculado. De esta forma, podemos decir que esta población puede semejarse a aquellas estudiadas a nivel mundial. Por las mismas consideraciones que se han mencionado solo nos atrevemos a decir que este sería un valor sugerido de prevalencia.

Debemos observar que sólo 18 niños (10.7\%) tuvieron diagnóstico previo de RA. Dentro del grupo de rinitis constituyen el $12.8 \%$, y en el grupo de RA el $19.6 \%$.

En todos los casos, es una prevalencia baja que realmente no representa la real magnitud del problema alérgico. Es probable que esto se deba a la presencia de una sintomatología muy leve en los pacientes, lo cual hace que los padres no le den la debida importancia. Tal vez se puede deber también a una evaluación médica inadecuada. Quizás, si estos niños tuvieran un tratamiento de ambas entidades, el número de crisis o exacerbaciones de asma sería mucho menor en cada uno de ellos. Otra posibilidad es que en realidad si se llegue a un diagnóstico adecuado en la consulta médica, pero por diferentes motivos no es comunicado adecuadamente a los padres o de repente no hayan comprendido la explicación de su médico. Esta misma cifra nos indica que el conocimiento de los padres sobre RA y su relación con el asma es deficiente.

Ante estos resultados, es importante tomar en cuenta que la coexistencia entre RA y asma si es frecuente en nuestro medio. Aunque no se haya establecido claramente el tipo de relación entre ellas, es necesario reconocerla en cada paciente para tener un mejor manejo.

En conclusión, la coexistencia de rinitis alérgica y asma bronquial es frecuente en nuestro medio. Es necesario reconocerla en cada paciente para tener un mejor manejo. El 10.7\% de niños que fueron evaluados tuvieron diagnóstico previo de RA, constituyendo un valor muy bajo a la cifra esperada. Esto revela que hay una falta de conocimiento e información de los padres sobre este tipo de alergia y su importante relación con el asma.

\section{Correspondencia :}

Pérez Lu, Julio Enrique.

Dirección: Las Orquídeas 315 Urb. La Molina Vieja, La Molina.

Teléfono: 495-2083, 495-3943, 9993-7336

Correo electrónico: julioepl1@ @otmail.com

\section{REFERENCIAS BIBLIOGRAFICAS}

1. Crystal-Peter BA J, Neslusan C, Crown W, Torres A. Treating allergic rhinitis in patients with comorbid asthma: The risk of asthma-related hospitalizations and emergency department visits. J Allergy Clin Immunol 2002; 109(1): 57-62.

2. Togias A. Systemic immunologic and inflammatory aspects of allergic rhinitis. J Allergy Clin Immunol. 2000; 106(5 Suppl):S247-50.

3. Leynaert B. Epidemiologic evidence for asthma and rhinitis comorbidity. J Allergy Clin Immunol, 2000;106 (5 Suppl):S201-5.

4. Fireman P. Therapeutic approaches to allergic rhinitis: Treating the child. J Allergy Clin Immun. 2000; 105(6 Pt 2):S616-21.

5. Corren J. 21st Century Management of upper respiratory allergic diseases: A focus on allergy and asthma - Day 1 , The impact of allergic rhinitis on bronchial asthma. J 
Allergy Clin Immunol 1998; 101(2):S352-S356.

6. Bousquet J, van Cauwenberge, Khaltaev N. Allergic rhinitis and its impact on asthma (ARIA). OMS. J Allergy Clin Immun 2001; 108(5):S147-S334.

7. Aguirre I; Carrasco C; Chiarella P. Normas y reglamentos para el manejo de asma en pediatría. Colegio Médico del Perú. 1997.

8. Ruiz-Gutiérrez F, Vega-Briceño L, Shion-Sam D, García Aguila A, Vargas-Castillo R, Chiarella-Ortigosa P. Prevalencia y presenctación estacional de rinitis alérgica en la población escolar del Distrito de Santiago de SurcoLima Perú. Bol Med Hosp Infant Mex 1997; 54: 276281.

9. The Internacional Study of Asthma and Allergies in Childhood (ISAAC) Steering Committee. Worldwide variation in prevalence of symptoms of asthama, allergic rhinoconjunctivitis, and atopic eczema: ISAAC. The Lancet 1998;351(9111):1220-1.

10.Mandl M, Nolop K, Lutsky B. Comparación de una sola administración diaria de furoato de mometasona $\left(\mathrm{NASONEX}^{\circledR}\right.$ ) y propionato de fluticasona en aerosol nasal acuoso para el tratamiento de la rinitis perenne. Ann Allergy Asthma Immunol 1997; 79: 370-8.

11.Badhwar A. y Druce H. Rinitis alérgica. Clínicas Médicas de Norteamérica 1992; 4:803-820.

12.Fireman P, Slavin R. Atlas de ALERGIA. Segunda edición. Ed. HARDCOURT-BRACE, España. 1997.

13.Naclerio R. Allergic Rhinitis. N Engl J Med 1991; 325(12):860-69.

14.Sociedad Mexicana de Rinología y Cirugía Facial. Rinología, ciencia y arte. MASSON-SALVAL Medicina, México. 1996.

15.Wright A; Holberg C, Martinez F, Halonen, Morgan W, Taussig. Epidemiology of Physician-Diagnosed Allergic Rhinitis in Childhood. Pediatrics 1994; 94(6 Pt 1):895901.

16.King H. Titulaciones cutáneas de punto óptimo: ¿son aún el estándar? Clínicas Otorrinolaringológicas de Norteamérica 1992;1:15-31.

17.Blaiss M. How to Determine the Cost-effectiveness of Available Allergic Rhinitis Treatments. Drug Benefit Trends 1998; 10(6):32-36.

18. Skoner D. Advances in allergic diseases: An update for the new millenium, Complications of allergic rhinitis. J1 of Allergy and Clinical Immunology 2000; 105(6):S605S609.

19.Santilli J; Nathan R; Glassheim J. Validation of the rhinitis outcomes questionnaire (ROQ). Ann Allergy Asthma Immunol 2001;86:222-225. 